\title{
DESIGNE AND CONSTRUCT AN AUTOMATIC HAND SANITIZER DISPENSER MACHINE
}

\author{
Sanket Jadhav \\ Department of Mechanical Engineering, \\ Zeal College of Engineering and Research Pune, Maharashtra, India
}

\begin{abstract}
Sanitizing hands is a simple act that pays in dividends when it comes to keeping ourselves healthy and safe. Hand sanitizing is also one of the key cornerstones of COVID- 19 prevention. Using a squeezing type sanitizer container spout in public places (hospitals, schools, shopping centers, industry plants and workplaces) is unsafe as it was touched by many hands. Therefore there is a need of automate the squeezing operation which guarantee a legitimate cleanliness and safe to use as touch less operation. Proposed unit is based on pre-programmed electronics and an inbuilt ultrasonic sensor. It detects hands when it comes in its programmed range and machine start pouring set amount of liquid into hand. Additionally there is preset delay is provided to serviceable and efficient use.
\end{abstract}

Keywords-COVID-19, Sanitizer, Automate, Ultrasonic sensor

\section{INTRODUCTION}

Cleaning or sterilizing an object or body part like hands or whole body known as sanitization. UV sanitization, Soap sanitization, alcohol sanitization, bleach sanitization are some of the common sanitization technique used in day-to-day life. Alcohol sanitization is the most effective way to sanitize hands since it is harmless on skin surface, vaporizes easily and kills most of the viruses, bacteria, and also removes dirt in our hands. To use any kind of liquid sanitizer we use storage container. It is required to touch the container In order to take some amount of sanitizer. Repeatedly touching the hand sanitizer containers to get a drop of sanitizer initiates contact with persons, which may be risky. Hence there is need for noncontact based hand sanitizer dispenser.

Therefore by utilizing ultrasonic sensor distance measuring capability, automatic hand sanitizer dispenser can give better solution for hygienic hand cleanliness. An automatic sanitizer dispenser unit can be installed in any area and effortlessly moved at any location as required.

\section{LITERATURE SURVEY}

In [1], the paper mention about the infection caused by drug resistant micro-organisms which causes increase in death rate and also complications, the multidrug resistant bacteria includes Methicillin Resistant Staphylococcus aureus, Extended Spectrum Beta-lactamase producing bacteria, Multidrug Resistant Pseudomonas aeruginosa, which are very common worldwide. Several antibiotics have increasing multidrug bacteria isolation rate, even personal protection equipment(PPE) can't be effective in isolation rate of MSRA. Hence they emphasize about the use of alcohol based hand sanitizers since the alcohol based hand sanitizers had negative association with MRSA isolation rate, which means that hand hygiene is very important in hospitals.

In [2], the paper says about the significance of hand washing with individual cleanliness. For ages, hand washing with cleanser and water has been viewed as a proportion of individual cleanliness. The idea of purging hands with a germicide specialist most likely rose in the mid nineteenth century. As ahead of schedule as 1822, a French drug specialist exhibited that arrangements containing chlorides of lime or soft drink could destroy the foul smells related with human bodies and that such arrangements could be utilized as disinfectants and sterilizers. In a paper distributed in 1825, this drug specialist expressed that doctors and different people going to patients with infectious illnesses would profit by soaking their hands with a fluid chloride arrangement.

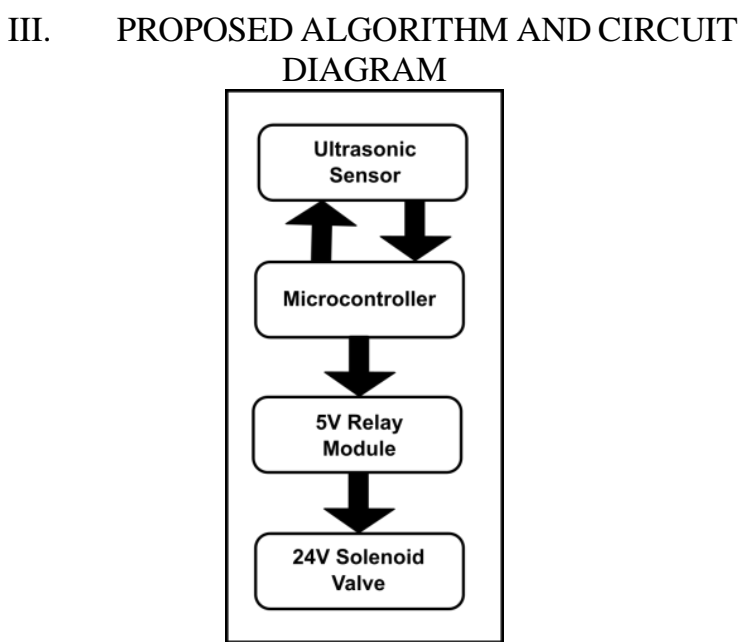

Figure 1. Proposed block diagram 


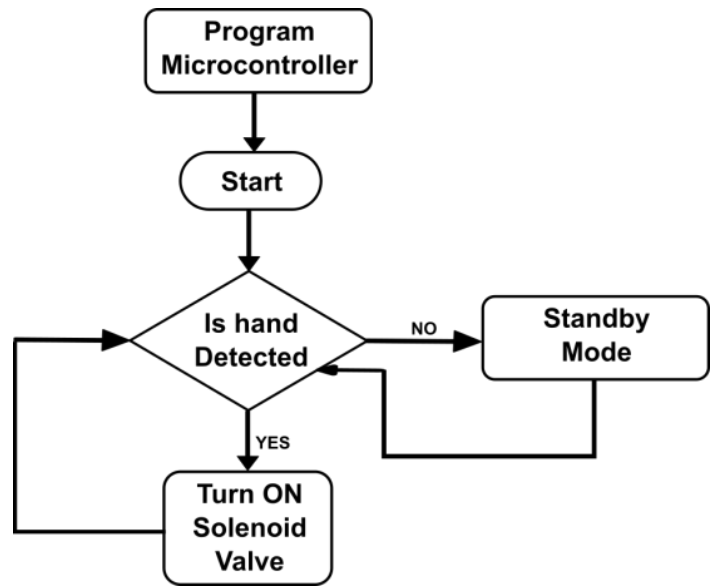

Figure 2. Flow chart of algorithm

The circuit diagram is shown in figure 3. Figure shows all electrical as well as mechanical connections. The microcontroller send pulses to sensor trigger pin and read echo pin. As an object detected sensor sends signal to microcontroller through echo pin. Then relay module is activating for three seconds which causes the opening of solenoid valve for three seconds. Therefore dispensing of sanitizer from container occurs.

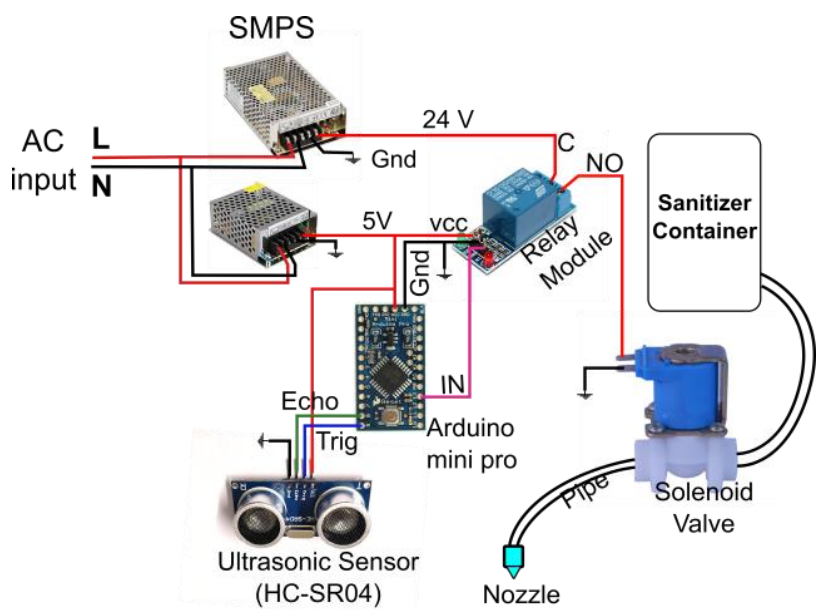

Figure 3. Circuit diagram

\section{COMPONENTS OF SANITIZER DISPENSER MACHINE}

\section{A. Arduino mini pro}

Atmel atmega 328p microcontroller is inbuilt in Arduino mini pro and it is programmed by arduino.cc software and arduino uno board. It is acting as a brain of the system. Digital pin 9 of microcontroller sends a signal to trigger pin of $\mathrm{HC}$ SR04 ultrasonic sensor and reading echo pin of the sensor through digital pin8. The distance is calculated by measuring the delay between triggering and getting feedback from echo pin.

Relay module is also controlled by the same microcontroller. As an object is detected in preprogramed range the digital pin 13 of the microcontroller send a signal to input (IN) pin of relay module.

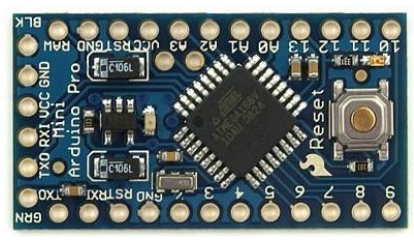

Figure 4. Arduino mini pro

\section{B. Ultrasonic Sensor}

HC-SR04 type ultrasonic sensor is used in this unit to detect object. It is powered by $5 \mathrm{v}$ power supply. The trigger and echo pin are connected to microcontroller in order to receive signal and send feedback. In proposed system it is power by 5 volt SMPS and trigger and echo pins are attach to digital pin 9 and 8 of microcontroller respectively. This sensor is capable to detect an object up to 4 meter away while in proposed system this set to detect an object (hand) within 15 $\mathrm{cm}$ from nozzle.

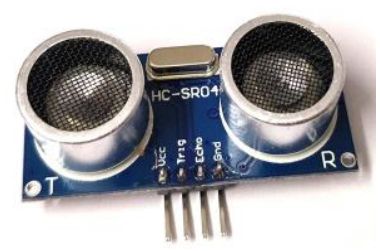

Figure 5. Ultrasonic sensor (HC-SR04)

\section{Relay Module}

The relay module is used to isolate two different voltage system from each other. This module is controlled by using input (IN) pin. As in circuit diagram shown this module is control by digital pin 13 of microcontroller additionally this is power by connecting VCC and GND pins to 5V SMPS.

Common (C) pin of relay is attach to positive of 24V SMPS and Normally Open (NO) pin is connect to one end of the solenoid valve

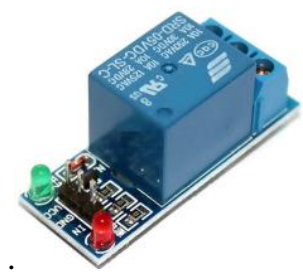

Figure 6. Relay module

\section{Solenoid Valve (SV)}

This is a valve operated by electromagnet. This electromagnet is activated by $24 \mathrm{~V}$ DC power supply. As electromagnet activate it allows the fluid to flow and vice a versa. One terminal of the SV is connected to NO terminal of relay module while other terminal is attach to negative of SMPS.

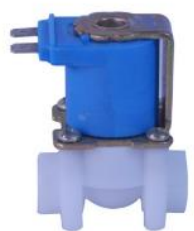

Figure 7. Solenoid valve 


\section{International Journal of Engineering Applied Sciences and Technology, 2021 \\ Vol. 5, Issue 10, ISSN No. 2455-2143, Pages 144-146 \\ Published Online February 2021 in IJEAST (http://www.ijeast.com)}

\section{E. SMPS}

SMPS (Switch mode power supply) is used to convert AC to DC. In proposed machine two SMPS are used. One of them provides $5 \mathrm{~V}$ DC output to power microcontroller, relay module and ultrasonic sensor. Another SMPS is used to power solenoid valve and it provide $24 \mathrm{~V}$ at output. Input of Both is connected to 230 volt AC.

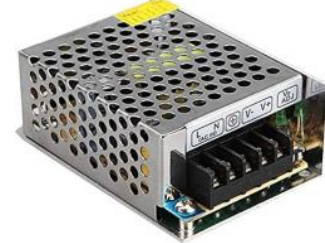

Figure 8. SMPS

\section{COSTING}

Table 1. Component list and their cost

\begin{tabular}{|c|l|c|}
\hline Sr.No. & Component name & Cost (Rs.) \\
\hline 1 & Arduino mini pro & $225 /-$ \\
\hline 2 & Ultrasonic sensor & $140 /-$ \\
\hline 3 & Relay Module & $100 /-$ \\
\hline 4 & Solenoid valve & $90 /-$ \\
\hline 5 & SMPS $(5 \mathrm{~V})$ & $150 /-$ \\
\hline 6 & SMPS $(24 \mathrm{~V})$ & $200 /-$ \\
\hline 7 & Pipe & $10 /-$ \\
\hline 8 & Three pin top & $25 /-$ \\
\hline 9 & Wire & $35 /-$ \\
\hline 10 & Sanitizer container $(21 t r)$. & $20 /-$ \\
\hline 11 & Casing \& Screws & $310 /-$ \\
\hline & Total & $1305 /-$ \\
\hline
\end{tabular}

Table 1 shows the list of components and total cost of the components including the manufacturing cost. Cost of proposed unit is in few thousands, whereas it can be further reduced, if machine is manufactured in batch and mass production.
VI.

\section{RESULT AND DISCUSSION}

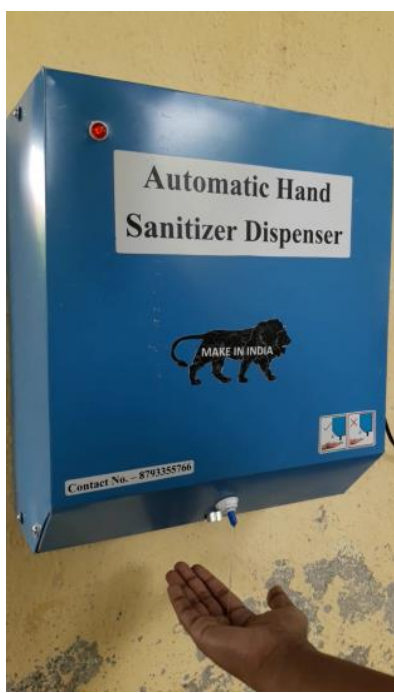

Figure 9. Proposed Automatic hand sanitizer dispenser machine (Wall mounted unit)

Place your hands below the bottom of the dispenser at the middle. Specified amount of sanitizer will be dispensed as soon as hand detected beneath the nozzle. Dispenser becomes ready for the next person quickly within 5 seconds.

\section{CONCLUSION}

The goal of this project is to ensure the maximum safety against the spread of germ causing by touching to the sanitizer container. The presented machine uses advanced technologies to achieve this goal.

This machine is cheapest compared to any other sanitization technique. Additionally, this dispenser is environment friendly since wastage from it is almost negligible. Also it can be refilled easily. Considering underprivileged section of society, this dispenser make affordable so that it available easily and can be used by everyone without any hassle.

\section{REFERENCES}

[1] Mitsuboshi S., and Tsugita M. (2018), Impact of alcoholbased hand sanitizers,antibiotic consumption, and other measures on detection rates of antibiotic resistant bacteria in rural Japaneshospitals, Journal of Infection and Chemotherapy, DOI: 10.1016/j.jiac.2018.08.013

[2] Boyce J., Pittet D. (2002), Guideline for Hand Hygiene in Health-Care Settings: Recommendations of the Healthcare Infection Control Practices Advisory Committee and the HICPAC/SHEA/APIC/IDSA Hand Hygiene Task Force, MMWR 2002; 51(No. RR-16).

[3] https://www.instructables.com/DIY-Easy-Non-ContactAutomatic-Hand-Sanitizer-Disp/

[4] https://www.instructables.com/Diy-Automatic-SanitizerDispenser/

[5] https://create.arduino.cc/projecthub/akshayjoseph666/cov id-19-automatic-hand-sanitizer-78cf6b

[6] https://create.arduino.cc/projecthub/search?q=sanitizer+d ispenser 